\title{
How Oceanic Vortices can be Super Long-Lived
}

\author{
G. G. Sutyrin \\ Graduate School of Oceanography, University of Rhode Island, Narragansett, Rhode Island, USA \\ घgutyrin@hotmail.com
}

Purpose. The article is aimed at substantiating theoretically amazing longevity (up to 5 years) of the individual vortices in the World Ocean despite strong fluctuations of the ocean currents and regardless of the Rossby wave dispersion properties.

Methods and Results. Evolution of the baroclinic vortices is considered in a hybrid two-layer ocean model over a topographic slope on the beta-plane. In the upper layer with strong potential vorticity anomalies, the currents are assumed to be balanced; in the lower layer at week potential vorticity anomalies, the currents are described in the traditional quasi-geostrophic approximation. Slow evolving almost circular vortices embedded in a vertically sheared current typical of the subtropical part of the ocean are described analytically. The theory shows how a baroclinic vortex is followed by the lee Rossby waves. The vortex drift across the mean current is caused mainly by the baroclinicdipole structure of the represented solution; while the vortex energy loss related to the Rossby wave radiation can be compensated by the energy stored in the mean currents.

Conclusions. The constructed model provides reasonable estimates of the energy drift and transfer typical of the ocean vortices with strong anomalies of potential vorticity. Direct support of long-lived vortices by the energy of the baroclinic mean flows irrespective of their stability, is of great importance for better understanding the physical mechanisms relating to significant longevity of the geophysical vortices and their movement.

Keywords: baroclinic vortices, sheared currents, Rossby waves

Acknowledgements: I would like to thank Gennady Korotaev, Gregory Reznik and Andrey Zatsepin for stimulating discussions and comments during the International Conference on Mesoscale and Submesoscale Processes in the Hydrosphere and Atmosphere (MSP-2018) Moscow, Russia. This study was supported by the USA National Science Foundation (grant OCE 1828843). I greatly appreciate useful comments of Ziv Kizner and anonymous reviewer.

For citation: Sutyrin, G.G., 2020. How Oceanic Vortices Can Be Super Long-Lived. Physical Oceanography, [e-journal] 27(6), pp. 677-691. doi:10.22449/1573-160X-2020-6-677-691

DOI: 10.22449/1573-160X-2020-6-677-691

(C) G. G. Sutyrin, 2020

(C) Physical Oceanography, 2020

\section{Introduction}

Long ago before satellite monitoring, strong fluctuations of ocean flows far from strong currents were discovered by the deployment of moored instruments and drifting floats during POLYGON-70 [1]. Swirls with spatial scales from tens to hundreds of kilometers and time scales from weeks to months were recognized to be the oceanic analog of synoptic storms in the atmosphere [2]. Such mesoscale variability is found ubiquitous in the most parts of the ocean, it contains more energy than any other form of motion [3]. Therefore, studying its governing dynamics became a central theme of physical oceanography for decades [4].

Modern observational programs and numerical modeling indicate that the kinetic energy of mesoscale variability is dominated by baroclinic vortices (called also eddies) with nearly vertical axis [5]. During their formation, mesoscale vortices are capable to trap water in their core and thus play a significant role in the transport and redistribution of water masses, heat, and biogeochemical properties throughout the oceans. They often persist for many rotational periods providing anomalous transport ISSN 1573-160X PHYSICAL OCEANOGRAPHY VOL. 27 ISS. 6 (2020) 
of fluid in their cores for thousands of kilometers [6]. Fig. 1 illustrates the records of evolution of ( $a$ ) the anticyclonic vortex for almost 5 years and $(b)$ the cyclonic vortex for 4.4 years (the blue, green, red, and yellow lines denote for December - January February, March - April - May, June - July - August, and September - October November, respectively, from [7]).
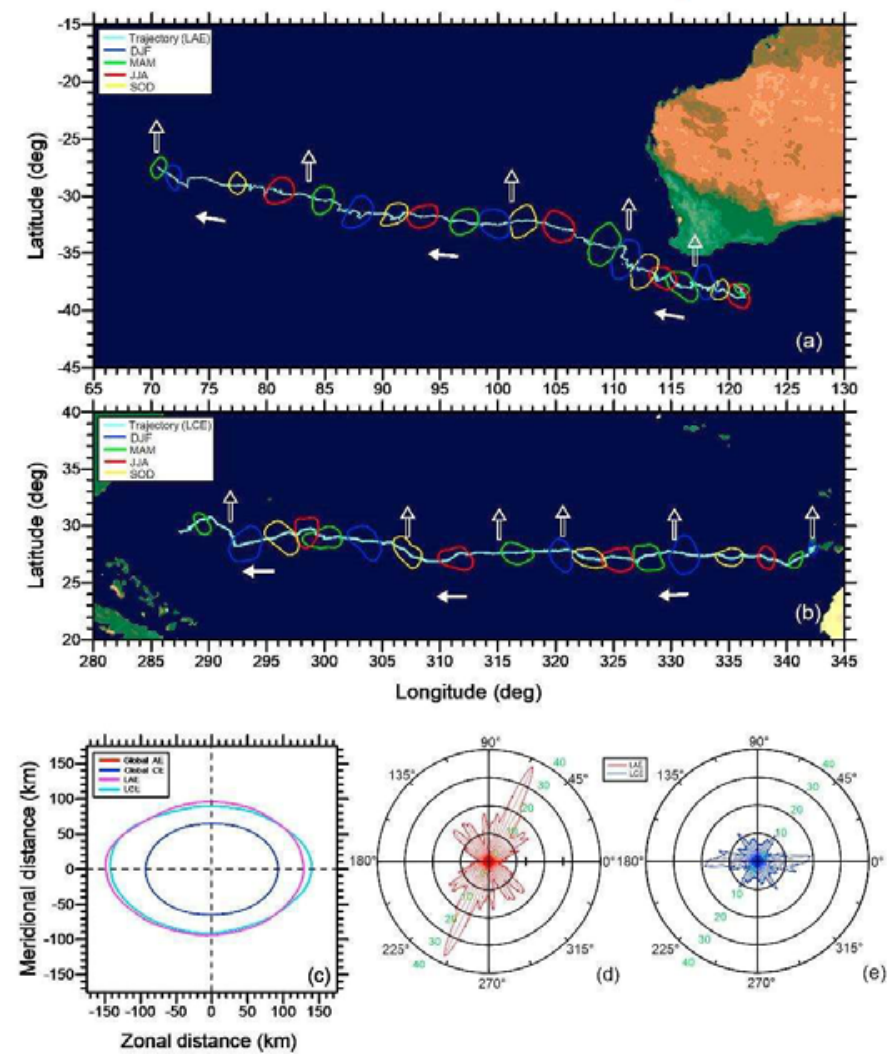

F i g. 1. "Evolutions of quarterly averaged contours of (a) the LAE (12/04/2003-06/03/2008, 1791 days) and (b) the LCE (12/01/2000-02/06/2004, 1604 days) during 1993-2016 (the blue, green, red, and yellow lines denote for December - January - February, March - April - May, June - July - August, and September - October - November, respectively). The light blue curves represent the trajectories of the two eddies, and the solid white arrows indicate their propagation directions. The vertical hollow white arrows indicate the locations where the eddy orientations start a new cycle of overall rotation. (c). The mean shapes of the LAE (the solid red line) and the LCE (the solid blue line) over their entire lifetimes. The globally averaged shapes of AEs (the dashed red line) and CEs (the dashed blue line) are also overlaid. (d) and (e) - Radar map of eddy orientation for the LAE (the red line) and the LCE (the blue line) ... Also overlaid are the timings of $0^{\circ}, 90^{\circ}, 180^{\circ}$, and $270^{\circ}$ orientations of the corresponding eddy (the blue, green, red, and yellow vertical dashed lines) ... (For interpretation of the references to color in this figure legend, the reader is referred to the web version of this article.)" [7, p. 85]

Amazing longevity of ocean vortices stimulated the development of several theoretical concepts to rationalize how they resist linear Rossby wave dispersion, related to significant background gradients of potential vorticity (PV), and survive in hostile turbulent environment; some of them have been summarized in books and reviews, e.g., [8-14]. The most popular class of horizontally localized nonlinear solutions to the equations of motion of rotating fluids at the beta-plane 
has been initiated by Stern [15] who introduced the term «modon», whereas steadily translating quasigeostrophic (QG) solutions were suggested by Larichev and Reznik [16]. Such modon-type steady propagating vortices were found analytically for various stratified models: essentially, they represent generalizations of self-propagating classical Lamb - Chaplygin dipole known for more than a century [17]. In all of them the resonance with Rossby waves is avoided by an active dipole inducing zonal propagation with the speed outside the range of linear Rossby waves (eastward or fast westward [18]).

Non-radiating monopolar anticyclones larger than the radius of deformation and with increased depth in their core were found in more general intermediate geostrophic models [19, 20] and rotating shallow-water models [21]. They sparked a great interest to the cyclone - anticyclones asymmetry which can be physically interpreted using a concept of local radius of deformation [22]. However, anticyclonic bias among long-lived eddies is not essential in the most parts of the ocean [23]. Additional physical mechanisms for the vortex intensification during the evolution of vortices with both polarities in homogeneous environment were also considered [24].

Generally, the drift velocity of real oceanic vortices has a significant component in the meridional direction which can be related to the Rossby wave radiation as demonstrated in several idealized models of radiating vortices at the beta-plane [25-28]. In the ocean at rest, nonlinear radiating vortices gradually decay, although at slower rate than the linear Rossby wave packet. This retardation of dispersion by nonlinearity is related to processes of axisymmetrization in fast rotating vortices [29, 30].

In reality, long-lived vortices are embedded in large-scale currents with the sloping isopycnals in the main thermocline storing roughly 1000 times more available potential energy (APE) than the kinetic energy (KE) associated with its thermal-wind current shear [31]. The opposite sign of background PV gradients, related to slopping isopycnals, ubiquitously leads to baroclinic instability [32]. The capability of large-scale APE to supply the eddy KE in vertically sheared flows is supported by an approximate agreement between the linear theory of baroclinic instability and observations [33]. The mesoscale vortices are, however, manifestly nonlinear, and coherent structures also emerge in many locations during the nonlinear evolution of the eddy field in numerical simulations demonstrating a near-universal tendency toward the production of grave vertical scales, with the barotropic and first baroclinic modes dominating almost everywhere [34]. Therefore, the two-layer models with bottom friction, playing an important quantitative role in the final scales and magnitude of eddies, are still frequently used [35]. In particular, the PV gradients in a stable flow cause vortex to remain more resilient to splitting, or shearing apart, of oceanic-like QG vortices by vertical shear flow in a $2 \frac{1}{2}$-layer model [36].

Recently it was found that the energy stored in flows with sloping isopycnals allows to compensate the Rossby wave dispersion for baroclinic vortices even in baroclinically stable background flows in the two-layer QG model [37]. When the background PV gradient in one layer is much smaller than in another layer, radiating vortices embedded in a marginally stable westward flow can be described as nearly steady. The theory was developed for nearly circular vortices coupled 
with the lee Rossby waves gaining their energy at the expense of APE in the mean flow without bottom friction. Moreover, competitive physical mechanisms which are responsible for an eddy meridional drift as well as the eddy self-intensification in baroclinically unstable flows with bottom friction were described analytically and confirmed numerically [37].

Here more general configurations of radiating vortices with strong PV anomalies are analyzed. The paper is organized as follows. First, we describe the two-layer hybrid model configuration and governing equations (Sec. 2). In Sec. 3, the solution is presented for the slowly evolving vortex coupled with Rossby waves associated with the PV gradient in the lower layer. In Sec. 4 this basic solution is used to provide explicit expressions for the wave drag and the meridional vortex drift at the larger time scale. In Sec. 5, we summarize results and draw conclusions.

\section{A hybrid two-layer model}

The two most important components of the ocean dynamics are low-frequency vortical motion and inertia-gravity waves. At the mesoscale and larger, the latter are relatively unimportant, and considered to be noise which have been causing headaches for modellers ever since Richardson's [38] pioneering forecast. Several different approaches have been used to construct balance models with reduced degrees of freedom by eliminating high-frequency oscillations from the governing equations (e.g., [39]). Such 'balanced' vortical dynamics is described by nonlinear advection of the potential vorticity (PV) and its subsequent inversion in order to obtain the flow velocity. For small PV anomalies from the background state, PV inversion has a simple linear form in widely used quasigeostrophic (QG) approximation (e.g., 40).

However, PV in the core of ocean eddies often strongly deviates from the background value. This will be taken into account in the upper layer of the twolayer model governing by the evolution of the PV in each layer [41]:

$$
\left\{\begin{array}{l}
\left(\frac{\partial}{\partial t}+U \frac{\partial}{\partial x}+V \frac{\partial}{\partial y}\right) \frac{f(y)+Z}{1+h / H}=0, \\
\frac{\partial Q}{\partial t}+J(\Psi, Q)+\mu_{0} \nabla^{2} \Psi=0, \\
Q=\nabla^{2} \Psi+f_{0} \frac{h+h_{b}}{H_{2}}+\beta y .
\end{array}\right.
$$

Here $\frac{f(y)+Z}{1+h / H}$ denotes PV in the upper layer with strong thickness anomaly, $h$, relative the mean thickness, $H ; Z=\frac{\partial V}{\partial x}-\frac{\partial U}{\partial y}$ - is the relative vorticity. In the deep lower layer with the mean thickness $H_{2}>>H$, the PV anomaly, $Q$, is described in the traditional QG approximation, $\Psi$ is the streamfunction, $\nabla^{2} \Psi-$ is 
the relative vorticity, $f=f_{0}+\beta y$ is the Coriolis parameter, $f_{0}$ its mean value, $\mu_{0}$ is the bottom drag coefficient and $h_{b}$ is the bottom topography, respectively.

Generally, the equation for the upper layer velocity can be written as

$$
(f+Z) U=-\frac{\partial B}{\partial y}-\frac{\partial V}{\partial t}, \quad(f+Z) V=\frac{\partial B}{\partial x}+\frac{\partial U}{\partial t}, \quad B=f_{0} \Phi+\frac{U^{2}+V^{2}}{2},
$$

where $B$ is the Bernoulli function and $f_{0} \Phi$ is the geopotential [42]. This form is useful to filter out inertia-gravity waves in balanced approximations of different accuracy for strong PV anomalies (see [43] and references therein). Finally, the upper geopotential and lower streamfunction are related by the quasistatic approximation

$$
\Phi=\Psi+g^{\prime} h / f_{0}, \quad g^{\prime}=g \frac{\Delta \rho}{\rho} .
$$

Note, that the equation for the mass balance in the upper layer follows from (1) and the vorticity equation obtained from (4)

$$
\frac{\partial Z}{\partial t}=-\frac{\partial(f+Z) U}{\partial x}-\frac{\partial(f+Z) V}{\partial y}, \quad \frac{\partial h}{\partial t}=\frac{\partial(H+h) U}{\partial x}-\frac{\partial(H+h) V}{\partial y} .
$$

Therefore, the sum of kinetic energy in the upper layer, $E_{1}=\rho \int(H+h)\left(B-f_{0} \Phi\right) d x d y$, the kinetic energy in the lower layer, $E_{2}=\frac{\rho H_{2}}{2} \int(\nabla \Psi)^{2} d x d y$, and the available potential energy (APE), $E_{p}=\frac{\rho g^{\prime}}{2} \int h^{2} d x d y$, is dissipated by the bottom friction

$$
\frac{d}{d t}\left(E_{1}+E_{2}+E_{p}\right)=-2 \mu_{0} E_{2} .
$$

The mean vertical shear is introduced by the laterally uniform zonal current described by the mean streamfuction $\bar{\Psi}=-U_{2} y$ in the lower layer, where uniform bottom slope $h_{b}=s y$. Besides the planetary beta, the variation of the Coriolis parameter, $\beta=\frac{d f}{d y}$, the background PV in each layer

$$
\left(f_{0}+\beta y\right) /(1+\alpha y / H), \bar{Q}=\beta_{2} y, \beta_{2}=\beta+f_{0} \frac{(s+\alpha)}{H_{2}}
$$

include the interface slope, $\alpha=f_{0} U_{2} / g$ '. Such basic state is known to become baroclinically unstable if PV gradients have opposite signs [44]. In the easterly shear, the slope is up towards the equator ( $\alpha>0$ in the Northern Hemisphere), actual upper layer mean flow is directed westward, while in the westerly shear, the slope is up towards the pole ( $\alpha<0$ in the Northern Hemisphere), actual upper layer mean flow is directed eastward [45]. In both hemispheres, the most long-lived 
eddies were recorded in subtropical corridors (Fig. 1) with typically easterly shear, where PV gradient (8) is reduced in the upper layer of the two-layer approximation.

Further, the perturbation streamfunction, , is introduced to be linearly related to deep PV anomaly,

$$
q=\nabla^{2} \Psi+F_{2}(\Phi-\Psi), F_{2}=\frac{f_{0}^{2}}{g^{\prime} H_{2}} .
$$

Then in the lower layer, the QG equation (2) takes the form

$$
J\left[\Psi+U_{2} y, q+\beta_{2} y\right]=-\frac{\partial q}{\partial t}-\mu_{0} \nabla^{2} \Psi .
$$

Multiplying (10) by $\mathrm{\rho H}_{2} \Psi$ and integrating, we see that instead of (7), the sum of kinetic energy in the upper layer, $E_{1}$, the perturbation kinetic energy in the lower layer, $\quad \tilde{E}_{2}=\frac{\rho H_{2}}{2} \int(\nabla \Psi)^{2} d x d y$, and the perturbation APE, $\tilde{E}_{p}=$ $=\frac{\rho g^{\prime}}{2} \int(h-\alpha y)^{2} d x d y$, can exchange with the mean energy

$$
\frac{d}{d t}\left(E_{1}+\tilde{E}_{2}+\tilde{E}_{p}\right)=W-2 \mu_{0} \tilde{E}_{2}, \quad W=-\rho U_{2} H_{2} \int q \frac{\partial \Psi}{\partial x} d x d y,
$$

where $W$ describes the meridional PV flux, serving as an energy source for perturbations in the vertically sheared flow (e.g., [46]). Usually, it is considered for baroclinic instability driven mostly by the release of mean APE when meridional PV gradients in the upper and lower layers have opposite signs [44]. However, (11) does not include the background PV gradients, therefore eddies have an ability to utilize the energy of a baroclinically stable mean flow, as we shall see in Section 4.

\section{Radiating vortex solution in marginally stable easterly shear}

Let's search for a quasi-steady solution, in the sense that the energy and the structure of the vortex change insignificantly during the characteristic time $T=L / U_{2}, L$ is the radius of the vortex core. We also neglect the bottom friction in (10). (However, having obtained the explicit solution, this result can then be used to evaluate r.h.s. in (10)). These assumptions will be verified afterwards.

Any functional dependence between $\Psi-U_{2} y$ and $q+\beta_{2} y$ eliminates l. h. s. in (10). An assumption that at the periphery $\Psi \rightarrow 0$ и $q \rightarrow 0$, implies the linear relationship in the lower layer

$$
q=-\beta_{2} \Psi / U_{2} \text { или } \nabla^{2} \Psi+F_{2}(\Phi-\Psi)+\beta_{2} \Psi / U_{2}=0 .
$$

In the upper layer with strong PV anomaly, $A=\frac{\beta y+Z-f_{0} h / H}{1+h / H} \sim f_{0}$ we can write

$$
\nabla^{2} \Phi-F_{1}(\Phi-\Psi)=A(1+h / H)-Z_{a}-\beta_{1} y=\Pi, F_{1}=\frac{f_{0}^{2}}{g^{\prime} H} .
$$


Here $Z_{a}=Z-\nabla^{2} \Phi$ is the ageostropic part of the vorticity included in $\Pi$, and $\beta_{1}=\beta-f_{0} \frac{\alpha}{H}$ is the far field PV gradient.

Focusing on the vicinity of marginal state with the easterly shear, $\left|\beta_{1}\right|<<\beta$, outside the vortex core we assume uniform $(A=0)$, then the thickness perturbation in (13) is compensated by the relative vorticity, while $\Pi=-Z_{a}$. For a given $\Pi(r)$ inside the core of nearly circular vortex, the solution of the coupled system (12) (13) can be found by decoupling into normal modes $\varphi_{j}=\Phi+\lambda_{j} \Psi$, as described by Sutyrin and Reznik [47] using QG model with topography without any mean flow. For each normal mode

$$
\left(\nabla^{2}-a_{j}^{2}\right) \varphi_{j}=\Pi, \quad a_{j}^{2}=F_{1}-\lambda_{j} F_{2}=F_{2}-\frac{F_{1}}{\lambda_{j}}-\frac{\beta_{2}}{U_{2}},
$$

where $\lambda_{j}$ are roots of the quadratic equation

$$
F_{1} \lambda^{2}-\left(F_{1}-F_{2}+\frac{\beta_{2}}{U_{2}}\right) \lambda-F_{1}=0
$$

Taking into account that in the marginal state $\beta_{1}=\beta-F_{1} U_{2}=0, \beta_{2}=L_{R}^{-2} U_{2}$, $L_{R}=\left(F_{1}+F_{2}\right)^{-1 / 2}$ is the baroclinic radius of deformation, the solution to (15) gives

$$
\begin{gathered}
a_{1}^{2}=-\frac{s f_{0}}{2 U_{2} H_{2}}+T, \quad a_{2}^{2}=-\frac{s f_{0}}{2 U_{2} H_{2}}-T, \\
T^{2}=\left(F_{1}+\frac{s f_{0}}{2 U_{2} H_{2}}\right)^{2}+F_{1} F_{2}=L_{R}^{-4}\left[(1-\chi+\widetilde{s})^{2}+\chi(1-\chi)\right] .
\end{gathered}
$$

Here $\widetilde{s}=\frac{s f_{0} L_{R}^{2}}{2 U_{2} H_{2}} \quad$ is proportional to the topographic slope $\chi=\frac{F_{2}}{F_{1}+F_{2}}=\frac{H}{H+H_{2}}<1$.

It can be shown from (17) that $a_{1}^{2}>0$ and $a_{2}^{2}=a_{1}^{2}-2 T=-\kappa^{2}<0$ for $\widetilde{s}>-1 / 2$. Then $\varphi_{1}(r)$ is the localized axisymmetric solution to (14) expressed by modified Bessel functions, while $\varphi_{2}(r, \theta)$ is the oscillating solution to (14) expressed using Bessel functions of the first and the second kind

$$
\begin{gathered}
\varphi_{1}=-K_{0}\left(a_{1} r\right) \int_{0}^{r} r^{\prime} d r^{\prime} I_{0}\left(a_{1} r^{\prime}\right) \Pi\left(r^{\prime}\right)-I_{0}\left(a_{1} r\right) \int_{r}^{\infty} r^{\prime} d r^{\prime} K_{0}\left(a_{1} r^{\prime}\right) \Pi\left(r^{\prime}\right), \\
\varphi_{2}=\frac{\pi}{2} Y_{0}(\kappa r) \int_{0}^{r} r^{\prime} d r^{\prime} J_{0}\left(\kappa r^{\prime}\right) \Pi\left(r^{\prime}\right)+\frac{\pi}{2} J_{0}(\kappa r) \int_{r}^{\infty} r^{\prime} d r^{\prime} Y_{0}\left(\kappa r^{\prime}\right) \Pi\left(r^{\prime}\right)+S,
\end{gathered}
$$




$$
\begin{gathered}
S=2 D \sum_{n=1}^{\infty} \frac{\cos (2 n-1) \theta}{2 n-1} J_{2 n-1}(\kappa r), \quad D=\int_{0}^{\infty} r^{\prime} d r^{\prime} J_{0}\left(\kappa r^{\prime}\right) \Pi\left(r^{\prime}\right), \\
\Phi=\frac{\lambda_{2} \varphi_{1}-\lambda_{1} \varphi_{2}}{\lambda_{2}-\lambda_{1}}=\frac{\left(\kappa^{2}+F_{1}\right)\left(\varphi_{1}-\varphi_{2}\right)}{2 T}+\varphi_{2}, \quad \Psi=\frac{\varphi_{1}-\varphi_{2}}{\lambda_{1}-\lambda_{2}}=F_{2} \frac{\varphi_{2}-\varphi_{1}}{2 T} .
\end{gathered}
$$

Here (19) corresponds to the lee Rossby waves which carries energy away from the eddy to the east. The last term, $S$, has to be added to satisfy the condition that no perturbations to the west $(x \rightarrow-\infty)$, as required without bottom friction [26]. Note, that it is defined by the integral value, $D$, in (20), which can be easily evaluated for uniform $\Pi$ inside the vortex core,

$$
\frac{D}{\Pi L^{2}}=\frac{J_{1}(\kappa L)}{\kappa L}
$$

The value $\frac{J_{1}(\kappa L)}{\kappa L}$ decreases from 0.5 for small $\kappa L<<1$, and becomes zero for $\kappa L \approx 3,8$; its characteristic value is 0.2 for $\kappa L=2.5$.

Without any topographic slope $(s=0) a_{1}^{2}=-a_{2}^{2}=L_{R}^{-2} \sqrt{1-\chi}$, as in [37]. The typical solution (21) with the Rossby wave wake over a flat bottom for the depth ratio $(\chi=1 / 8)$ is shown at Fig. 2 for uniform $\Pi$ inside the vortex core. The amplitudes of the upper layer cyclone and the lower layer anticyclone at the right panels for the large vortex $\left(L=3 L_{R}\right)$ are slightly larger than at the left panels for the medium vortex $\left(L=2 L_{R}\right)$. In both cases, the lower layer patterns look similar to the upper panel in Fig. 4 plotted by Flierl [26, p. 53] for the solidbody rotating core inside bowl-shaped $h$ in the limit of deep lower layer. Note an eastward shift of the anticyclone center in the lower layer relative the cyclone center in the upper layer resulting in its poleward drift to be evaluated in the next section.


F i g. 2. Solution (21) for the medium $\left(L=2 L_{R}\right)$ and large $\left(L=3 L_{R}\right)$ vortices 


\section{Estimates of the PV flux and the meridional drift}

In the marginally stable easterly shear, the solution (18) - (21) represents lee Rossby waves generated by an upper layer vortex which is stationary at the time scale $T$. Far from the vortex center to the east,

$$
\varphi_{2} \approx \pi D Y_{0}(\kappa r) \sim \sqrt{\frac{2 \pi}{\kappa r}} \cdot D \sin \left(\kappa r-\frac{\pi}{4}\right) \text { для }-\frac{\pi}{2}<\theta<\frac{\pi}{2}, \quad \kappa r>>1,
$$

which can be used to estimate the net PV flux in (11), using (12) and (21)

$$
W=\rho \beta_{2} H_{2} \lim _{r \rightarrow \infty} r \Psi^{2}=\frac{\pi \rho \beta_{2} H_{2}}{\kappa}\left(\frac{F_{2} D}{2 T}\right)^{2}>0 .
$$

We see that vortices of both signs extract energy from the linearly stable mean vertical shear while generating the lee Rossby waves. Thus, $W$ can be interpreted as a compensation to the form drag exerted by the Rossby waves (see [26]) which is inversely proportional to the lower layer thickness in (24). Assuming it is balanced by the bottom friction according to (11), the energy in the presence of bottom friction in the lower layer can be estimated as $\widetilde{E}_{2}=W / 2 \mu_{0}$. Without a topographic slope in the QG limit, (24) is equivalent to (20) in [35] that was obtained for the upper layer PV flux and confirmed in their numerical simulations.

The solution (18) - (21) can be also utilized to evaluate the characteristic meridional drift of the vortex related to the asymmetric part of (19), defined by $S(r, \theta)$. The upper layer meridional velocity at the vortex center is defined by the first azimuthal mode of $\varphi_{2}$, described by $2 D x J_{1}(\kappa r) / r$ in (20), so that

$$
\begin{gathered}
V_{0}=\frac{\partial \Phi}{\partial x}=\varepsilon \frac{\partial S}{\partial x}=\varepsilon \kappa D=\varepsilon \Pi L J_{1}(\kappa L), \\
\varepsilon=1-\frac{\kappa^{2}+F_{1}}{2 T}=\frac{1}{2}\left(1-\frac{1-\chi+\widetilde{s}}{T L_{R}^{2}}\right)=\frac{1}{2}\left(1-\frac{1-\chi+\widetilde{s}}{\sqrt{(1-\chi+\widetilde{s})^{2}+\chi(1-\chi)}}\right)>0 .
\end{gathered}
$$

The coefficient $\varepsilon \sim \frac{\chi}{4(1+\widetilde{s})^{2}}<<1$ for the small depth ratio $\chi$, that validates the quasi-steady solution (18) - (21). The velocity of the meridional drift (25) is proportional to the vortex intensity and corresponds to the vortex polarity: cyclones with positive $\Pi$ drift poleward, while anticyclones with negative $\Pi$ drift equatorward. From (26) we see that the positive topographic slope reduces the meridional drift, while the negative topographic slope leads to larger meridional drift than over a flat bottom.

When nonzero PV gradient in the upper layer is taken into account, both zonal and meridional vortex translation have additional components due to the development of the beta-gyres, analyzed in several studies (e.g., [30, 48] and references therein). This leads also to a gradual change of the vortex intensity due to the Lagrangian conservation of PV at the vortex center in the upper layer. Therefore, in the baroclinically unstable mean flow $\left(\beta_{1}<0\right)$ the vortex can intensify due to the meridional drift induced by the lee Rossby waves [37]. 
Let's consider typical vortex parameters for the anticyclone and the cyclone shown in Fig. 1. The anticyclone was crossing the subtropical south Indian Ocean along a major east-west pathway of long-lived eddies with typical parameters summarized in Table 1 [49, p. 5412]. The azimuthal velocity $V_{m} \sim 0,25 \mathrm{~m} \cdot \mathrm{s}^{-1}$ at the radius of the vortex core $L \sim 60 \mathrm{~km}$ corresponds to the geopotential anomaly at the vortex center $f_{0} \Phi_{c}=f_{0} L V_{m} \sim 1 \mathrm{~m}^{2} \cdot \mathrm{s}^{2}$ with $f_{0} \sim 7 \cdot 10^{-5} \mathrm{~s}^{-1}$. This value can be related to the relative stretching by introducing the characteristic gravity wave speed, $v_{g}=\sqrt{g^{\prime} H}$, to be interpreted within an equivalent-barotropic approximation [50]

$$
f_{0} \Phi_{c}=v_{g}^{2} h_{c} / H
$$

From the vertical scale of velocity decay at their Fig. 9 [49, p. 5419], the thickness of the upper layer $H \sim 0,35 \mathrm{~km}$ together with the reduced gravity $g^{\prime} \sim 0,01 \mathrm{~m} \cdot \mathrm{s}^{-2}$ gives $v_{g} \sim 1,8 \mathrm{~m} \cdot \mathrm{s}^{-1}$ и $L_{R}=v_{g} / f_{0} \sim 26 \mathrm{~km}$, which is slightly less than a half of the vortex core radius. Then $h_{c} / H \sim 1 / 3$, so that $\Pi \sim-f_{0} / 2$, while the PV anomaly $A \sim-f_{0} / 3$.

In order to have zero PV gradient at the periphery in the upper layer owing to the compensation of the planetary PV gradient $\beta=2 \cdot 10^{-11} \mathrm{~m}^{-1} \cdot \mathrm{s}^{-1}$, the mean interface slope $\alpha=\beta H / f_{0} \sim 10^{-4} \quad$ corresponds to the vertical shear $U_{2} \sim g^{\prime} \alpha / f_{0} \sim 0,03 \mathrm{~m} \cdot \mathrm{s}^{-1}$, which agrees with the mean westward drift of the anticyclone shown in Fig. 1 during 5 years. For small $\chi \kappa \sim L_{R}^{-1}$, $|D| \sim 0,1 f_{0} L^{2} \sim 2,5 \cdot 10^{4} \mathrm{~m}^{2} \cdot \mathrm{s}^{-1}$, then the rate of the energy transfer from the mean flow into lee Rossby waves can be estimated from (24) as

$$
W=\frac{\pi \chi}{4 L_{R}} \rho U_{2} H D^{2} \sim 20 M W .
$$

The equatorward drift of such anticyclone given by $V_{0} \sim \frac{\Pi L}{100} \sim 0,02 \mathrm{~m} \cdot \mathrm{s}^{-1}$, has to be reduced by $2 / 3$ after an adjustment to the nearly steady state with bottom friction as shown in numerical simulations by Sutyrin and Radko [37]. This agrees with the observed equatorward $\operatorname{drift} \sim U_{2} / 5$, of the anticyclone evaluated from its path at Fig. 1.

The parameters of the cyclone drifting westward for 4.4 years in the Canary corridor at Fig. 1 are similar to the anticyclone in the South Indian Ocean [51]. However, the observed poleward drift of this cyclone was much slower than for above considered anticyclone indicating contributions of other effects, e.g., the beta-gyres related to the negative PV gradient in the upper layer $\left(\beta_{1}<0\right)$, which are able to compensate the poleward drift induced by the lee Rossby waves.

\section{Conclusions}

This study generalizes an analytical QG model [37] to a hybrid two-layer model for a circular vortex with strong PV anomaly imbedded in the vertical shear 686

PHYSICAL OCEANOGRAPHY VOL. 27 ISS. 6 (2020) 
over a topographic slope. The analytical tractability is achieved by focusing on the limit in which background flow is marginally stable with respect to baroclinic instability. In this regime, the solution at the advective time scale represents a stationary pattern, consisting of the circular vortex in the upper layer, accompanied by the Rossby wave wake it continuously generates. This configuration represents an example in which the energy required to generate and maintain the wake pattern is supplied entirely by the stable background flow. The release of energy stored in zonal large-scale currents is traditionally attributed to the action of baroclinic instability. However, the considered case represents an alternative mechanism for the energy baroclinic cascade towards mesoscale. The estimations of the meridional drift for realistic ocean vortices indicate that it is much smaller than the shear velocity, validating the assumptions of the theory. This theory adds an important brick into a wall of important physical mechanisms helping long-lived eddies to survive in a turbulent ocean which are summarized below.

Using barotropic (2D) models is a common first step to evaluate coherent eddies in large-scale flows [52]. Inviscid decay (Landau damping) of asymmetric perturbations to stable vortices is responsible for the process of vortex axisymmetrication [53] and the energy transfer from a weak satellite at the periphery of a strong vortex due to curved stretching out [54]. These simple theories rationalize peripheral amplification of 2D vortices in simulations by [55].

Monopolar baroclinic vortices of medium size are known to be able to remain baroclinically stable (e.g., [56]) in contrast to jet currents over a flat bottom which always have opposite PV gradients in the upper and lower layer [57]. The growth of perturbations and the puzzling survival of wide baroclinic eddies in predominantly monopolar states strongly depend on the initial vortex structure and the model stratification (e.g., [58]). In a three-layer model, the stabilizing effect of a middle layer with uniform PV owing to a weakening of the vertical coupling between the upper and lower layers and a reduction of the PV gradient in the deep layer at rest has been revealed by [59]. Nonlinear saturation of baroclinic instability in the form of tripolar states plays an important role in survival of the long-lived vortices [60].

The background lateral shear and strain are known to provide an effective mechanism for gradual erosion of the stable vortices due to vorticity stripping at the core boundary studied mostly in 2D flows [61-63]. The similar vortex erosion is expected in baroclinic vortices embedded in the large-scale Rossby wave, where the role of available potential energy for the vortex resistance remains underinvestigated [64]. A substantial amplification of fluid rotation due to radial redistribution of the angular momentum during erosion of the vortex core was demonstrated assuming the momentum mixing is proportional to the strain at the periphery of circular vortices [65].

In summary, among several known physical mechanisms helping coherent eddies to be super long-lived in the ocean, the essential role is played by the energy transfer from the large-scale vertically sheared currents described in the QG model [37] and for more general hybrid model in this paper. Its efficiency is proportional to the vertical shear and the vortex intensity squared, i.e., it does not depend on the vortex polarity. This conclusion is supported by examples of the extreme PHYSICAL OCEANOGRAPHY VOL. 27 ISS. 6 (2020) 
longevity and long-distance propagation of coherent cyclones and anticyclones consistently found in the subtropical eddy corridors with the easterly shear (e.g., Fig. 1). Further studies are in progress.

\section{Acknowledgements}

I would like to thank Gennady Korotaev, Gregory Reznik and Andrey Zatsepin for stimulating discussions and comments during the International Conference on Mesoscale and Submesoscale Processes in the Hydrosphere and Atmosphere (MSP-2018) Moscow, Russia. This study was supported by the USA National Science Foundation (grant OCE 1828843). Useful comments of Ziv Kizner and an anonymous reviewer are greatly appreciated.

\section{REFERENCES}

1. $\quad$ Brekhovskikh, L.M., Fedorov, K.N., Fomin, L.M., Koshlyakov, M.N. and Yampolsky, A.D., 1971. Large-Scale Multi-Buoy Experiment in the Tropical Atlantic. Deep Sea Research and Oceanographic Abstracts, 18(12), pp. 1189-1206. https://doi.org/10.1016/00117471(71)90026-X

2. Koshlyakov, M.N. and Monin, A.S., 1978. Synoptic Eddies in the Ocean. Annual Review of Earth and Planetary Sciences, 6, pp. 495-523. https://doi.org/10.1146/annurev.ea.06.050178.002431

3. Robinson, A.R., Ed., 1983. Eddies in Marine Science. Berlin: Springer-Verlag, 612 p. doi:10.1007/978-3-642-69003-7

4. Koshlyakov, M.N. and Belokopytov, V.N., 2020. Mesoscale Eddies in the Open Ocean: Review of Experimental Investigations. Physical Oceanography, 27(6), pp. 559-572. doi:10.22449/1573-160X-2020-6-559-572

5. McWilliams, J.C., 2008. The Nature and Consequences of Oceanic Eddies. In: M. W. Hecht, H. Hasumi, Eds., 2008. Ocean Modeling in an Eddying Regime. Washington, DC: American Geophysical Union, pp. 5-15. doi:10.1029/177GM03.

6. Dong, C., McWilliams, J.C., Liu, Y. and Chen, D., 2014. Global Heat and Salt Transports by Eddy Movement. Nature Communications, 5, 3294. https://doi.org/10.1038/ncomms4294

7. Chen, G., Han, G. and Yang, X., 2019. On the Intrinsic Shape of Oceanic Eddies Derived from Satellite Altimetry. Remote Sensing of Environment, 228, pp. 75-89. https://doi.org/10.1016/j.rse.2019.04.011

8. McWilliams, J.C., 1985. Submesoscale, Coherent Vortices in the Ocean. Reviews of Geophysics, 23(2), pp. 165-182. doi:10.1029/RG023i002p00165

9. Flierl, G.R., 1987. Isolated Eddy Models in Geophysics. Annual Review of Fluid Mechanics, 19, pp. 493-530. https://doi.org/10.1146/annurev.fl.19.010187.002425

10. Korotaev, G.K., 1988. Theoretical Modeling of Synoptic Activity of an Ocean. Kiev: Naukova Dumka, 157 p. (in Russian).

11. Korotaev, G.K., 1997. Radiating Vortices in Geophysical Fuid Dynamics. Surveys in Geophysics, 18(6), pp. 567-618. doi:10.1023/A:1006583017505

12. Nezlin, M.V. and Sutyrin, G.G., 1994. Problems of Simulation of Large, Long-Lived Vortices in the Atmospheres of the Giant Planets (Jupiter, Saturn, Neptune). Surveys in Geophysics, 15(1), pp. 63-99. doi:10.1007/BF00665687

13. Carton, X., 2001. Hydrodynamical Modeling of Oceanic Vortices. Surveys in Geophysics, 22(3), pp. 179-263. doi:10.1023/A:1013779219578

14. Sokolovskiy, M.A. and Verron, J., 2014. Dynamics of Vortex Structures in a Stratified Rotating Fluid. Cham: Springer, 382 p. doi:10.1007/978-3-319-00789-2

15. Stern, M.E., 1975. Minimal Properties of Planetary Eddies. Journal of Marine Research, 33(1), pp. 1-13. Available at: http://images.peabody.yale.edu/publications/jmr/jmr33-0101.pdf [Accessed: 20.09.2020]. 
16. Larichev, V. and Reznik, G., 1976. On Two-dimensional Solitary Rossby Waves. Doklady Akademii Nauk SSSR, 231(5), pp. 1077-1079. Available at: http://www.mathnet.ru/links/ab484331932b9f8f6f361e722e3cb8e3/dan40813.pdf [Accessed: 15 October 2020] (in Russian).

17. Meleshko, V.V. and van Heijst, G.J.F., 1994. On Chaplygin's Investigations of TwoDimensional Vortex Structures in an Inviscid Fluid. Journal of Fluid Mechanics, 272, pp. 157-182. https://doi.org/10.1017/S0022112094004428

18. Kizner, Z., Berson, D., Reznik, G. and Sutyrin, G., 2003. The Theory of the Beta-Plane Baroclinic Topographic Modons. Geophysical and Astrophysical Fluid Dynamics, 97(3), pp. 175-211. doi:10.1080/0309192031000108706

19. Mikhailova, E.I. and Shapiro, N.B., 1980. Two-Dimensional Model of Synoptic Disturbances Evolution in the Ocean. Izvestiya Akademii Nauk SSSR. Fizika Atmosfery i Okeana, 16(8), pp. 823-833 (in Russian).

20. Petviashvili, V.I., 1980. Red Spot of Jupiter and the Drift Soliton in a Plasma. JETP Letters, 32(11), pp. 619-622. Available at: http://www.jetpletters.ac.ru/cgibin/articles/download.cgi/1434/article_21816.pdf [Accessed: 30 October 2020].

21. Sutyrin, G.G., 1985. On the Theory of solitary anticyclones in a rotating liquid. Doklady Akademii Nauk SSSR, 280(5), pp. 1101-1105 (in Russian).

22. Sutyrin, G.G. and Yushina, I.G., 1989. Numerical Modelling of the Formation, Evolution, Interaction and Decay of Isolated Vortices. In: J. C. J. Nihoul, B. M. Jamart, Eds., 1989. Mesoscale/Synoptic Coherent Structures in Geophysical Turbulence. Elsevier, pp. 721-736. doi:10.1016/S0422-9894(08)70217-4

23. Chelton, D.B., Schlax, M.G. and Samelson, R.M., 2011. Global Observations of Nonlinear Mesoscale Eddies. Progress in Oceanography, 91(2), pp. 167-216. doi:10.1016/j.pocean.2011.01.002

24. Sutyrin, G.G., 2018. Super Long-Lived Ocean Eddies. In: A. G. Zatsepin, A. I. Ginzburg, A. G. Kostianoy, S. A. Sviridov, Eds., 2018. Proceedings of the International Symposium "Mesoscale and Submesoscale Processes in the Hydrosphere and Atmosphere" (MSP-2018), 30 October - 02 November 2018, Moscow. Moscow: SIO RAS, pp. 46-47. Available at: https://ocean.ru/phocadownload/MSP-2018/msp_2018.pdf [Accessed: 20.09.2020] (in Russian).

25. Korotaev, G.K., 1980. Structure, Dynamics and Energetic of Synoptic Variability of the Ocean. Preprint. Sevastopol: Marine Hydrophysical Institute, 64 p. (in Russian).

26. Flierl, G.R., 1984. Rossby Wave Radiation from a Strongly Nonlinear Warm Eddy. Journal of Physical Oceanography, 14(1), pp. 47-58. https://doi.org/10.1175/15200485(1984)014<0047:RWRFAS>2.0.CO;2

27. McDonald, N.R., 1998. The Decay of Cyclonic Eddies by Rossby Wave Radiation. Journal of Fluid Mechanics, 361, pp. 237-252. doi:10.1017/S0022112098008696

28. Nycander, J., 2001. Drift Velocity of Radiating Quasigeostrophic Vortices. Journal of Physical Oceanography, 31(8), pp. 2178-2185. https://doi.org/10.1175/15200485(2001)031<2178:DVORQV>2.0.CO;2

29. Sutyrin, G.G., 1987. The Beta Effect and the Evolution of a Localized Vortex. Doklady Akademii Nauk SSSR, 296(5), pp. 1076-1080. Available at: http://www.mathnet.ru/links/bb945265df7fd03879fae801ee00058a/dan48090.pdf [Accessed: 30 October 2020].

30. Kravtsov, S. and Reznik, G., 2019. Numerical Solutions of the Singular Vortex Problem. Physics of Fluids, 31(6), 066602. https://doi.org/10.1063/1.5099896

31. Gill, A.E., Green, J.S.A. and Simmons, A.J., 1974. Energy Partition in the Large-Scale Ocean Circulation and the Production of Mid-Ocean Eddies. Deep Sea Research and Oceanographic Abstracts, 21(7), pp. 499-528. https://doi.org/10.1016/0011-7471(74)90010-2

32. Vallis, G.K., 2006. Atmospheric and Oceanic Fluid Dynamics: Fundamentals and LargeScale Circulation. Cambridge: Cambridge University Press, 2006. 866 p. 
33. Ferrari, R. and Wunsch, C., 2009. Ocean Circulation Kinetic Energy: Reservoirs, Sources, and Sinks. Annual Review of Fluid Mechanics, 41, pp. 253-282. https://doi.org/10.1146/annurev.fluid.40.111406.102139

34. Venaille, A., Vallis, G.K. and Smith, K.S., 2011. Baroclinic Turbulence in the Ocean: Analysis with Primitive Equation and Quasigeostrophic Simulations. Journal of Physical Oceanography, 41(9), pp. 1605-1623. doi:10.1175/JPO-D-10-05021.1

35. Radko, T., Peixoto de Carvalho, D. and Flanagan, J., 2014. Nonlinear Equilibration of Baroclinic Instability: The Growth Rate Balance Model. Journal of Physical Oceanography, 44(7), pp. 1919-1940. https://doi.org/10.1175/JPO-D-13-0248.1

36. Vandermeirsh, F., Morel, Y. and Sutyrin, G., 2002. Resistance of a Coherent Vortex to a Vertical Shear. Journal of Physical Oceanography, 32(11), pp. 3089-3100. https://doi.org/10.1175/1520-0485(2002)032<3089:ROACVT>2.0.CO;2

37. Sutyrin, G.G. and Radko, T., 2021. Why the Most Long-Lived Eddies are Found in the Subtropical Ocean Westward Flows. Ocean Modelling, 153, 101825.

38. Richardson, L.F., 1922. Weather Prediction by Numerical Process. Cambridge: The University Press, 258 p. Available at: https://archive.org/details/weatherpredictio00richrich [Accessed: 30 October 2020].

39. Monin, A.S., 1972. Weather Forecasting as a Problem in Physics. M. I. T. Press, 199 p.

40. Kamenkovich, V.M., Koshlyakov, M.N. and Monin, A.S., eds., 1986. Synoptic Eddies in the Ocean. Netherlands, Dordrecht: Springer, 1986. 443 p.

41. Sutyrin, G.G. and Grimshaw, R., 2005. Frictional Effects on the Deep-flow Feedback on the $\beta$-Drift of a Baroclinic Vortex over Sloping Topography. Deep Sea Research Part I: Oceanographic Research Papers, 52(11), pp. 2156-2167. doi:10.1016/j.dsr.2005.06.017

42. Sutyrin, G.G., 1986. Synoptic Movements of Finite Amplitude. Doklady Akademii Nauk SSSR, 290(5), pp. 1084-1088. Available at: http://www.mathnet.ru/links/5662892d8fad089be8eb8a118be68902/dan47702.pdf [Accessed: 15 October 2020] (in Russian).

43. Sutyrin, G.G., 2004. Agradient Velocity, Vortical Motion and Gravity Waves in a Rotating Shallow-Water Model. Quarterly Journal of the Royal Meteorological Society, 130(601), pp. 1977-1989. https://doi.org/10.1256/qj.03.54

44. Phillips, N.A., 1954. Energy Transformations and Meridional Circulations Associated with Simple Baroclinic Waves in a Two-Level, Quasi-geostrophic Model. Tellus, 6(3), pp. 274286. https://doi.org/10.3402/tellusa.v6i3.8734

45. Tulloch, R., Marshall, J., Hill, C. and Smith, K.S., 2011. Scales, Growth Rates, and Spectral fluxes of Baroclinic Instability in the Ocean. Journal of Physical Oceanography, 41(6), pp. 1057-1076. https://doi.org/10.1175/2011JPO4404.1

46. Thompson, A.F. and Young, W.R., 2006. Scaling Baroclinic Eddy Fluxes: Vortices and Energy Balance. Journal of Physical Oceanography, 36(4), pp. 720-738. https://doi.org/10.1175/JPO2874.1

47. Reznik, G.M. and Sutyrin, G.G., 2001. Baroclinic Topographic Modons. Journal of Fluid Mechanics, 437, pp. 121-142. doi:10.1017/S0022112001004062

48. Sutyrin, G.G. and Flierl, G.R., 1994. Intense Vortex Motion on the Beta Plane: Development of the Beta Gyres. Journal of the Atmospheric Sciences, 51(5), pp. 773-790. https://doi.org/10.1175/1520-0469(1994)051<0773:IVMOTB>2.0.CO;2

49. Dilmahamod, A.F., Aguiar-González, B., Penven, P., Reason, C.J.C., De Ruijter, W.P.M., Malan, N. and Hermes, J.C., 2018. SIDDIES Corridor: A Major East-West Pathway of Long-Lived Surface and Subsurface Eddies Crossing the Subtropical South Indian Ocean. Journal of Geophysical Research: Oceans, 123(8), pp. 5406-5425. https://doi.org/10.1029/2018JC013828

50. Sutyrin, G.G., 2020. How Baroclinic Vortices Intensify Resulting from Erosion of Their Cores and/or Changing Environment. Ocean Modelling, 156, 101711. https://doi.org/10.1016/j.ocemod.2020.101711 
51. Pegliasco, C., Chaigneau, A. and Morrow, R., 2015. Main Eddy Vertical Structures Observed in the Four Major Eastern Boundary Upwelling Systems. Journal of Geophysical Research: Oceans, 120(9), pp. 6008-6033. https://doi.org/10.1002/2015JC010950

52. Burgess, B.H., Dritschel, D.G. and Scott, R.K., 2017. Vortex Scaling Ranges in TwoDimensional Turbulence. Physics of Fluids, 29(11), 111104. https://doi.org/10.1063/1.4993144

53. Sutyrin, G.G., 1989. Azimuthal Waves and Symmetrization of an Intense Vortex. Doklady Akademii Nauk SSSR, 304(5), pp. 1086-1091. Available at: http://www.mathnet.ru/links/8a31183a00e5c592d7d0ebfc624dda46/dan48505.pdf [Accessed: 30 October 2020] (in Russian).

54. Sutyrin, G.G., 2019. On Vortex Intensification due to Stretching out of Weak Satellites. Physics of Fluids, 31(7), 075103. https://doi.org/10.1063/1.5098068

55. Sutyrin, G.G. and Radko, T., 2019. On the Peripheral Intensification of Two-Dimensional Vortices in Smaller-Scale Randomly Forcing Flow. Physics of Fluids, 31(10), 101701. https://doi.org/10.1063/1.5118752

56. Benilov, E.S., 2004. Stability of Vortices in a Two-Layer Ocean with Uniform Potential Vorticity in the Lower Layer. Journal of Fluid Mechanics, 502, pp. 207-232. doi:10.1017/S0022112003007547

57. Sutyrin, G., 2015. Generation of Deep Eddies by a Turning Baroclinic Jet. Deep Sea Research Part I: Oceanographic Research Papers, 101, pp. 1-6. doi:10.1016/j.dsr.2015.02.011

58. Sutyrin, G.G. and Radko, T., 2016. Stabilization of Isolated Vortices in a Rotating Stratified Fluid. Fluids, 1(3), 26. doi:10.3390/fluids1030026

59. Sutyrin, G., 2015. Why Compensated Cold-Core Rings Look Stable. Geophysical Research Letters, 42(13), pp. 5395-5402. doi:10.1002/2015GL064378

60. Sutyrin, G.G., 2016. On Sharp Vorticity Gradients in Elongating Baroclinic Eddies and Their Stabilization with a Solid-Body Rotation. Geophysical Research Letters, 43(11), pp. 58025811. doi:10.1002/2016GL069019

61. Legras, B. and Dritschel, D., 1993. Vortex Stripping and the Generation of High Vorticity Gradients in Two-Dimensional Flows. Applied Scientific Research, 51(1-2), pp. 445-455. https://doi.org/10.1007/BF01082574

62. Mariotti, A., Legras, B. and Dritschel, D.G., 1994. Vortex Stripping and the Erosion of Coherent Structures in Two-Dimensional Flows. Physics of Fluids, 6(12), 3954. https://doi.org/10.1063/1.868385

63. Legras, B., Dritschel, D.G. and Caillol, P., 2001. The Erosion of a Distributed TwoDimensional Vortex in a Background Straining Flow. Journal of Fluid Mechanics, 441, pp. 369-398. https://doi.org/10.1017/S002211200100502X

64. Sutyrin, G. and Carton, X., 2006. Vortex Interaction with a Zonal Rossby Wave in a QuasiGeostrophic Model. Dynamics of Atmospheres and Oceans, 41(2), pp. 85-102. doi:10.1016/j.dynatmoce.2005.10.004

65. Sutyrin, G.G., 1992. Maintenance of Quick Fluid Rotation in the Cores of Long-Lived Oceanic Eddies. Journal of Marine Systems, 3(6), pp. 489-496. doi:10.1016/09247963(92)90019-5

About the author:

Georgy G. Sutyrin, Emeritus Senior Scientist, Graduate School of Oceanography, University of Rhode Island (Narragansett, Rhode Island, 02882, USA), Dr. Sci. (Phys.-Math.), ORCID ID: 00000002-5222-7800, gsutyrin@hotmail.com

The author has read and approved the final manuscript.

The author declares that he has no conflict of interest. 